\title{
EL PROCESO DE GESTIÓN EMOCIONAL: INVESTIGACIÓN, APLICACIÓN Y EVALUACIÓN
}

\author{
Núria García Blanc, Raquel Gomis Cañellas, Agnés Ros Morente y Gemma Filella Guiu \\ Universidad de Lleida, España \\ Universidad de Barcelona, España
}

\section{Resumen}

El presente artículo tiene como objetivo presentar las aportaciones del GROP, Grupo en Investigación Psicopedagógica, en el campo de la educación emocional durante los últimos diez años. Este grupo de investigación está integrado por profesorado de la Universidad de Lleida (UdL) y la Universidad de Barcelona (UB). En primer lugar, se presenta el modelo teórico de resolución de conflictos que consta de 4 pasos: El primer paso es la conciencia emocional, que incluye la comprensión y legitimación de las emociones; el segundo paso se centra en la reducción de la intensidad emocional y la impulsividad; el tercer paso sería el uso de diferentes estrategias de regulación de las emociones, y el cuarto y último paso incluye asertividad comunicación. Dicho modelo se ha gamificado en forma de varios videojuegos para, posteriormente, aplicarse en contextos reales y analizar sus resultados. Además, este artículo también recoge otras aportaciones relevantes diseñadas por este grupo como son el modelo de las 5 competencias emocionales y los instrumentos CDE-A, CDE-R, Secundaria CDE-SEC y CDE-9-13.

Palabras clave: Educación emocional, Resolución de conflictos, Gamificación, Convivencia

\section{Abstract}

The present article aims to present the contributions of the research group named GROP Grupo en Investigación Psicopedagógica, in the field of emotional education during the last ten years. The members of this research group are from the University of Lleida (UdL) and the University of Barcelona (UB). In the first place, the theoretical model of conflict resolution is presented. The model is divided into 4 phases. The first step is emotional awareness, which includes the understanding and legitimation of emotions; the second step focuses on the reduction of emotional intensity and impulsivity; the third step would be the use of different emotion regulation strategies, and the fourth and final step includes assertive communication. This model has been gamified in several video games. Thereafter, it has been applied in real contexts and its results have been analysed. In addition, this article also collects other relevant contributions designed by this research group, such as the model of the 5 emotional competencies and the instruments CDE-A, CDE-R, Secundaria CDE-SEC and CDE-913.

Keywords: Emotional education, Conflict resolution, Gamification, Coexistence

\section{Gemma Filella Guiu \\ gemma.filella@udl.cat}

Departamento de Pedagogía, Facultad de Educación, Psicología y Trabajo social Universidad de Lleida 


\section{Introducción}

Como es sabido, los seres humanos somos seres sociales, pues, vivimos en comunidad y por el hecho de convivir, es frecuente que surjan conflictos. La causa de éstos puede ser variada: intereses, necesidades, identidades, valores u opiniones dispares (Díez, 2004; Jares, 2006; Jeong, 2008; Rozenblum, 2007). En los centros educativos, dicha convivencia también genera conflictos. Algunos datos reflejan que el $90 \%$ de los estudiantes de educación secundaria obligatoria (E.S.O) presencian conflictos o los altos índices de episodios violentos y agresión: uno de cada cuatro niños ha experimentado bullying en la escuela (Gabarda, 2014; Save the Children, [STC], 2015) y el $45 \%$ de los alumnos se identifican como víctimas de una situación violenta vivida en su centro escolar (Dinkes, Kemp, Baum y Synder, 2009).

Existe un acuerdo científico en señalar que estas agresiones no se deben a un alto nivel de hostilidad, sino a la falta de estrategias y habilidades para resolver dichos conflictos de manera eficaz (Ortega, 2010). Es decir, una falta de competencias emocionales de los implicados (Bisquerra, 2008, 2014; Ceballos, Correa, Correa, Rodríguez, Rodríguez, y Vega, 2010; Extremera y Fernández-Berrocal, 2003; Jiménez Benedit, 2008; Romera, Rodríguez y Ortega, 2015; entre otros).

El papel que desempeñan las emociones cuando se presenta un conflicto es fundamental en palabras de Gross y Jazaieri (2014), así como con el comportamiento (Koole, 2009; Redorta, Obiols y Bisquerra, 2006). De hecho, algunos estudios afirman que cuando las emociones afloran en un contexto inapropiado de manera muy intensa o por un periodo muy prolongado de tiempo, pueden llegar a ser problemáticas (Gross, 2015; Gross y Jazaieri, 2014; Werner y Gross, 2010).

Por este motivo, reconocer, controlar y manejar las emociones se convierte en un factor clave para asegurar una interacción sana entre iguales y la disminución de comportamientos violentos y agresivos (Filella, Cabello, PérezEscoda y Ros-Morente, 2016; Pérez-Escoda, Filella, Soldevila y Fondevila, 2013; Romera et al., 2015). Precisamente, existe un alto grado de correlación entre una elevada inteligencia emocional y la calidad de las relaciones interpersonales (Extremera y Fernández Berrocal, 2004a; Extremera y Fernández Berrocal, 2004b).

Precisamente, se ha demostrado que un clima de centro positivo, se traduce en una mayor participación y motivación de los alumnos. De esta manera, se advierte una mejora en el proceso de aprendizaje aumentando la autoestima de los estudiantes, así como sus habilidades sociales y un ambiente más enriquecedor (Álvarez-García, Dobarro, Rodríguez, Núñez y Álvarez, 2013).
Por el contrario, se ha comprobado que cuando dicho clima de aula es negativo, el centro escolar deviene más vulnerable para sufrir casos de bullying (Wang, 2014). A su vez, el rendimiento académico o el bienestar de los alumnos puede verse afectado y la víctima puede percibir el centro como amenazante y violento o llegar a desarrollar graves síntomas de ansiedad (Caballo, Arias, Calderero, Salazar y Irurtia, 2011; Pérez, Gázquez, Mercader, Molero y García, 2001).

De esta manera, poner en marcha un proceso de aprendizaje centrado en desarrollar las habilidades y el manejo de las emociones, los conflictos cotidianos servirán como base para aprender nuevas estrategias de resolución, respuestas para solucionar conflictos futuros y desarrollarse socialmente (Villamediana, Donado y Zerpa, 2015).

\section{Modelo para la resolución de conflictos interpersonales}

Dada la relevancia de los beneficios de una convivencia positiva y la adecuada gestión de los conflictos, el GROP desarrolló, después de una intensa investigación, de más de 10 años, un modelo teórico centrado en la regulación emocional y la resolución de conflictos de manera asertiva y basado en sus propias investigaciones además de los hallazgos de otros destacados investigadores, como Gross (2007). Este modelo consta de cuatro pasos:

\section{Paso 1. Conciencia emocional}

La conciencia emocional puede definirse como la habilidad de reconocer, identificar y comprender las emociones propias y ajenas, saber ponerles nombre y tomar conciencia de la interacción que existe entre emoción, cognición y comportamiento (Altarriba y Bauer, 2004; Bisquerra, 2009). Ésta incluye la comprensión emocional y la legitimidad emocional. La primera es la capacidad de comprender las emociones de los demás, en la que la empatía juega un papel fundamental y la segunda es la tolerancia y el respeto por las emociones de los demás, lo cual ayuda a reducir la ansiedad en situaciones de conflicto (Bisquerra, 2009; File1la, 2014, Redorta et al., 2006).

Para ello, el manejo y la comprensión de vocabulario emocional resultan imprescindibles. ya que conocer el vocabulario emocional, aumenta a su vez, el mundo emocional del individuo y su correcta interpretación (Bisquerra, 2000).

De acuerdo con Thompson, Dizén y Berenbaum (2009) la atención a la emoción y la claridad de la emoción cobran gran importancia dentro de la conciencia emocional. El primer término informa sobre cómo la persona nota, piensa y monitorea su estado de ánimo mientras que la segunda hace referencia a la claridad de cómo la persona entiende sus propias emociones, las discrimina y etiqueta. Algunos estudios también han demostrado que cuanto más claro tenga una persona qué está sintiendo, menor variabilidad emocional presenta. Esto se puede conseguir con una actitud introspectiva y mediante la reflexión sobre nuestras 


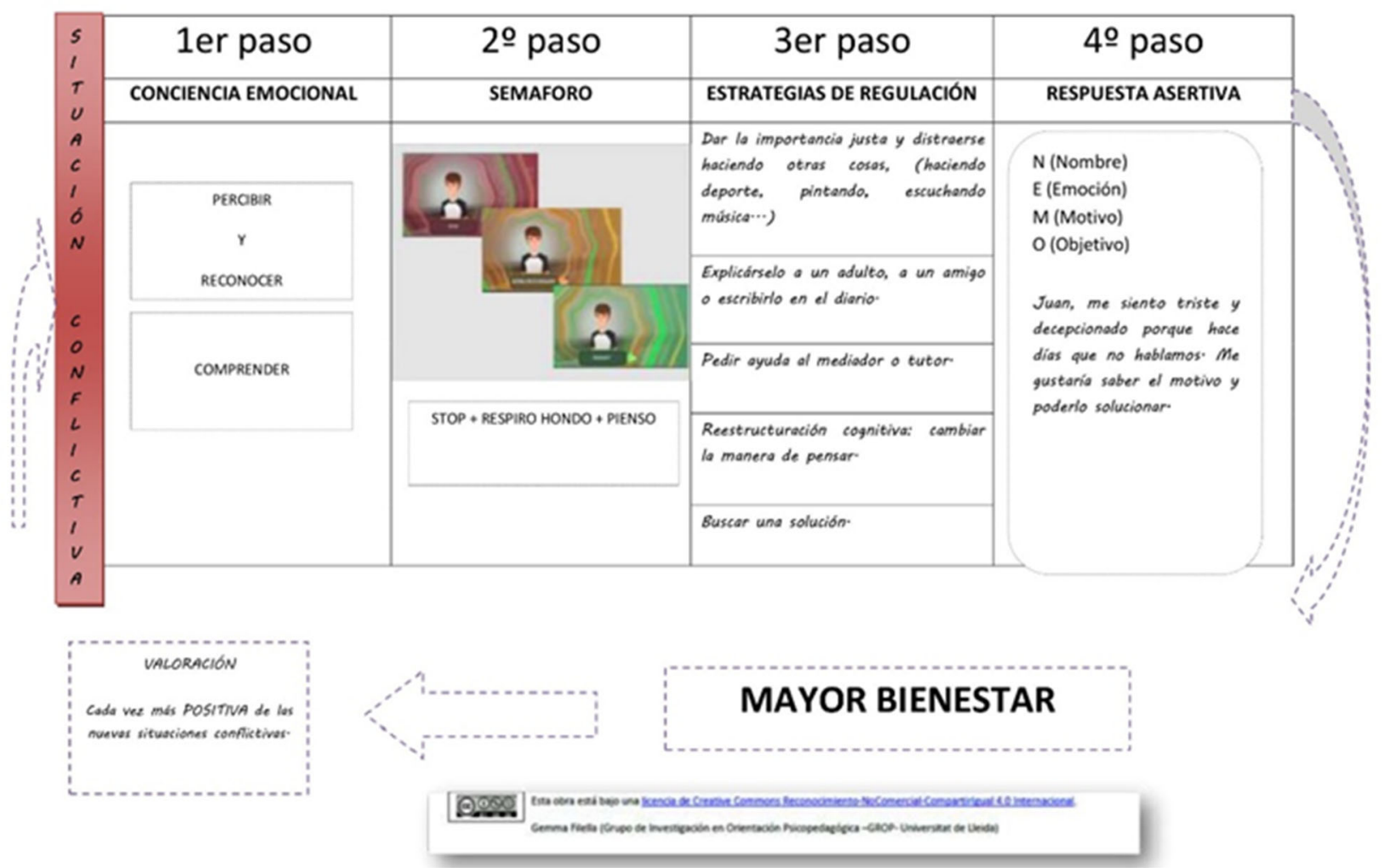

Figura 1.

Proceso de regulación emocional. Fuente: Filella, 2016

experiencias para saber reconocerlas como experiencias emocionales (Stegge y Meerum, 2007). Ya que, si no somos conscientes de nuestras emociones y, por ende, de la información que estas nos dan sobre cómo estamos y cuáles son nuestras metas, necesidades y preocupaciones, no sabremos cómo regularlas correctamente (Thompson Dizén y Berenbaum, 2009).

\section{Paso 2. Parar, reducir la impulsividad e intensidad emocional}

Después de ser capaz de tomar conciencia de las emociones: saber qué se siente, por qué y legitimar sus propias emociones, el segundo paso se centra en reducir la intensidad de las emociones negativas mediante una pausa, con el fin de evitar dejarse llevar por las emociones negativas y cometa acciones de las que después pueda arrepentirse.

Según Wittmann y Paulus (2008), la impulsividad afecta negativamente a la capacidad de concentrarse en pensamientos significativos, a la planificación de las acciones y de objetivos. Incluso algunos estudios señalan que la impulsividad puede afectar las habilidades de razonamiento cognitivo (Grant, Odlaug y Schreiber, 2012). Por lo tanto, para avanzar en la resolución de conflictos, se hace imprescindible rebajar la intensidad de las emociones y así adqui- rir una nueva perspectiva, más manejable y con menor impulsividad. Para lograr rebajar la intensidad de las emociones y su estado de activación fisiológica, trabajar la conciencia emocional o reflexionar. Se pueden utilizar técnicas de relajación y respiración (Bisquerra, 2009). Entre ellas, existe la técnica del semáforo (Goleman, 1995).

\section{Paso 3. Estrategias de regulación emocional}

El tercer paso consiste en incorporar las estrategias de manejo emocional cuyo objetivo es, siguiendo a Gross (2007), establecer una serie de parámetros y pasos para aprender competencias emocionales desde edades muy tempranas y así poder manejar la conflictividad mejor y aumentar el bienestar social e individual. Así pues, este paso incorpora las siguientes estrategias:

En el primer bloque encontramos las estrategias centradas en evitar la reactividad/impulsividad centradas en el control de la reacción emocional en un primer momento.

La primera de ellas, la expresión emocional, o la necesidad de comunicar una experiencia emocional significativa con las personas del entorno, el mismo día que ocurre, pasado un tiempo; una vez o diversas veces, de forma repetitiva (Zech y Rimé, 2005). Por otro lado, expresión emocional 
Tabla 1.

Estrategias de regulación emocional. Fuente: elaboración propia.

\begin{tabular}{|c|l|}
\hline \multirow{2}{*}{$\begin{array}{c}\text { ESTRATEGIAS CENTRADAS } \\
\text { EN EVITAR LA REACTIVIDAD/ } \\
\text { IMPULSIVIDAD }\end{array}$} & La expresión emocional \\
\cline { 2 - 2 } & La expresión emocional verbal \\
\cline { 2 - 2 } & La expresión emocional escrita \\
\hline \multirow{2}{*}{ ESTRATEGIAS CENTRADAS } & Listracción \\
\cline { 2 - 2 } EN LOS ANT la silla vacia \\
\cline { 2 - 2 } & Reevaluar una situación \\
\cline { 2 - 2 } & Buscar una solución \\
\hline
\end{tabular}

verbal obliga a pasar las emociones a sentimientos a través del lenguaje. Esto posibilita que se lleve a cabo un distanciamiento progresivo de la experiencia emocional, al desvanecimiento de ésta y en consecuencia a la recuperación emocional (Rimé, 2009).

La expresión emocional escrita surge como alternativa a la expresión emocional verbal en caso que la persona no pueda comunicarse verbalmente ya sea porque no sabe cómo hacerlo o porque expresar sus emociones está mal visto culturalmente (Butler, Lee y Gross, 2007; Lu y Stanton, 2010). Hay principalmente dos maneras de trabajar dicha estrategia: la escritura positiva (Suhr, Risch y Wilz, 2017) o la escritura de sucesos traumáticos, significativos o estresantes (Lu y Stanton, 2010). Algunos estudios demuestran que, gracias a esta estrategia, se reducen los síntomas físicos, las visitas a centros sanitarios y bajas laborales disminuyen (Baikie y Wilhelm, 2005), se reducen síntomas depresivos como la rumiación y la supresión expresiva (Gortner, Rude y Pennebacker, 2006), y, a la vez, aumenta la sensación de bienestar (Baikie y Wilhelm, 2005).

Dentro de este último bloque encontramos la Técnica de la silla vacía que consiste en imaginar que en la silla vacía que hay enfrente, está la persona con la que se tiene dificultades para poder así practicar la expresión verbal: cómo se siente, que necesidades tiene, qué quiere decirle (Conoley, Conoley, McConnell y Kimzey,1983; Narkiss-Guez, Enav, Guez y Diamond, 2014) así como anticiparse a la situación real e imaginarse la respuesta y su reacción (Elliott y Greenberg, 2016). Otra de las finalidades de esta técnica es tratar temas pendientes y no resueltos (Elliott y Greenberg, 2016; Wagner-Moore, 2004).

El segundo bloque pertenece a las estrategias centradas en los antecedentes donde se recogen las estrategias de distracción y las de reevaluación.

En primer lugar, encontramos la distracción que se utiliza para desconectar de las emociones negativas, actuando como filtro para bloquear la información emocional antes de que pueda ser evaluada y posibilitando la modulación de estímulos emocionales de alta intensidad (Sheppes, Scheibe, Suri, Radu, Blechert y Gross, 2014). Ya que, dejar de pensar en una situación o una emoción negativa ayuda a evitar la rumiación (Augustine y Hemenover, 2008) y tiene un efecto positivo y significativo en la regulación emocional (Webb, Miles y Sheeran, 2012).

La estrategia de reevaluar la situación ha demostrado ser una de las estrategias más eficaces tanto a nivel afectivo, como cognitivo y social, pues disminuye la experiencia y la expresión emocional negativa (Gyurak, Gross y Etkin, 2011; John y Gross, 2004; McRae, Ciesielsky y Gross, 2012). Ésta, ayuda a enfocar el conflicto desde otro ángulo para poder encontrar una solución. También se ha observado un mejor funcionamiento interpersonal, más probabilidades de ser queridos por los demás y un mayor bienestar personal (Gross y John, 2003; Gross, 2002, 2014; Gyurak, Gross y Etkin, 2011). Esta estrategia goza de una cierta dificultad, pero las personas que la utilizan se caracterizan por mantener una alta autoestima, así como una actitud 
optimista ante las presiones. Son capaces de reinterpretarlas y trabajar activamente para reparar las emociones negativas generadas por una situación estresante que sienten, pero, a su vez, son capaces de expresar sus emociones positivas y negativas socialmente (Gross y John, 2004).

Por último, la última estrategia refiere a buscar una solución si un suceso nos hace sentir ansiedad o malestar. Además, se debe proporcionar herramientas para que sea más fácil resolver dicha situación. Un ejemplo de ello es el modelo, ya clásico, de Krumboltz (1979) que tiene como pasos: i) definir el problema, ii) establecer un plan, iii) identificar alternativas, iv) evaluación de uno mismo, v) investigar posibles resultados, vi) eliminar alternativas, vii) experimentar.

\section{Paso 4. Comunicación y conducta asertiva}

Bien es sabido que hay muchas maneras de enfrentarse a un conflicto. Entre todos los modelos propuestos, Caballo (1997) presentó un modelo bidimensional que se caracteriza por la expresión humana (de manera manifiesta o encubierta) y la forma de expresarse (usando el castigo y la amenaza, o no). Con esta combinación se clasifican cuatro tipos de conductas, recogidas y definidas por otros autores a posteriori (Monjas y González, 2000; Roca, 2014):

El primer tipo es la conducta pasiva entendida como aquellas situaciones en que la persona no expresa, o no se atreve a expresar, de forma adecuada sus pensamientos, sentimientos y lo que quiere, con la tendencia a hacer lo que otros les dicen para evitar deteriorar sus relaciones. Prefieren no enfrentarse a los conflictos y no tienen en cuenta sus propios derechos ni deseos.

Con una conducta agresiva se realizan comportamientos de castigo o amenazantes y no se respetan los derechos, deseos y sentimientos de los demás. A su vez, para solucionar los conflictos utilizan la imposición y el autoritarismo.

Las personas con una conducta asertiva son capaces de comunicarse expresando adecuadamente sus sentimientos, pensamientos e intereses respetando los de los demás. Quiere conseguir sus objetivos conservando sus relaciones interpersonales y favoreciendo la generación de emociones positivas. Este tipo de conductas facilitan la resolución de conflictos, pues, fomentan el respeto y una buena comunicación.

En una conducta pasivo-agresiva, se combinan las características de las conductas pasivas y agresivas: la persona no se expresa y realiza conductas de castigo y amenaza.

La estrategia que se presenta a continuación "NEMO", fomenta la comunicación asertiva y se trabaja desde la comunicación positiva, la cual va dirigida a mejorar el bienestar psicológico independientemente de la razón por la cual se están comunicando y a resolver los conflictos sin dañar la integridad ni la autoestima de las personas (MuñízVelázquez y Álvarez-Nobell 2013).

Se empieza pronunciando el nombre de la persona implicada en el conflicto, a continuación, se verbaliza la emoción que le está generando conjuntamente con el motivo. Finalmente, se comunica al interlocutor cuál es el objetivo de la conversación. Las siglas de esta estrategia se corresponden con: $\mathrm{N}$ (Nombre de la persona con la que se está hablando) + E (la emoción que te ha generado) + M (el motivo por el cual te ha generado esta emoción) $+\mathrm{O}$ (con qué objetivo te estas comunicando con él o ella).

\section{La gamificación del modelo para la resolución de con- flictos inter-personales: Los videojuegos Happy 8-12 y Happy 12-16}

Este modelo teórico expuesto anteriormente se ha gamificado en forma de varios videojuegos: Happy 8-12 (para los alumnos de segundo y tercer ciclo de educación primaria), Happy 12-16 (para los estudiantes de la Educación Secundaria Obligatoria), Happy Sports y Happy Adultos. Además, está en proceso de elaboración el videojuego Happy for Families orientado a las familias.

Todos incluyen un software especializado en el trabajo de las competencias emocionales, siguiendo el modelo de Bisquerra y Mateo (2019). Ambos softwares son videojuegos diseñados específicamente para que los niños y adolescentes aprendan a gestionar mejor sus competencias emocionales y de este modo, puedan responder a los conflictos que puedan surgir en su vida diaria (Webster-Stratton y Reid, 2004) de manera asertiva y con un mayor bienestar, que en largo plazo ofrece muchas ventajas en términos de bienestar y adaptabilidad al contexto.

Ambos videojuegos siguen los mismos pasos, pero contienen situaciones acordes a cada grupo de edad. Todos ellos presentan 25 conflictos, 15 de los cuales se desarrollan en el contexto escolar y 10 entre hermanos en el contexto familiar, a excepción de Happy for Families y Happy Adultos que tienen 30. A modo de ejemplo se muestran dos de ellos. En el contexto escolar: "Nuria es una niña que dice mentiras para tener amigas" y en el contexto familiar: "Tu hermano necesita concentrarse para poder estudiar para un examen, pero pones música muy fuerte". Ante el conflicto y dependiendo de la situación que se encuentre, el alumno deberá elegir entre tres tipos de respuesta: respuesta agresiva, respuesta pasiva o respuesta asertiva. Ésta última es el único tipo de respuesta que cuenta como correcta.

Asimismo, cabe señalar que el jugador no siempre se encuentra bajo el mismo rol, sino que existe la posibilidad de ser acosador, víctima u observador. Este hecho resulta totalmente relevante debido a que ayuda a promover la cantidad de posibilidades y estrategias que los estudiantes deberán aprender para llevar a cabo una respuesta adecuada. 


\section{Otras aportaciones del GROP}

\section{Aplicación y resultados de los videojuegos}

Hasta la fecha, se han realizado varios estudios piloto que demuestran que el modelo de resolución de conflictos tiene cierta eficacia. El primer software que se aplicó y evaluó fue el videojuego Happy 8-12 en varios centros de educación primaria pertenecientes a la ciudad de Lleida (España), cuya muestra sumaba 576 alumnos. El procedimiento se concreta en un diseño pre-post con grupo control. Los resultados arrojan que la práctica de resolución de conflictos con el software Happy 8-12 mejora las competencias emocionales y reducen los niveles de ansiedad de los alumnos de manera estadísticamente significativa $(\mathrm{p}<.001$ en ambos casos). De todos modos, es preciso mencionar que el efecto del aprendizaje fue discreto, pues, variables como las competencias emocionales y el clima social del aula requieren de un tiempo prolongado y un entrenamiento más duradero que un curso académico. Actualmente se está implementando.

Como sucedió con los resultados de los estudiantes de educación primaria, se llevó a cabo un estudio piloto con un diseño pre-post en una muestra de 903 estudiantes de educación secundaria. Los resultados de dicha investigación demuestran que los alumnos que participaron en la formación con el software gamificado presentaron una tendencia significativa a mejorar sus competencias emocionales ( $p$ $<.001)$.

En el presente estudio se advirtió el mismo fenómeno que en el estudio con los alumnos de educación primaria, pues, el efecto en el proceso de aprendizaje en las habilidades emocionales fue discreto $\mathrm{y}$ donde se advierte el mayor efecto es en la escala de ansiedad $(0,2$ a 0,4$)$. Esto se explica por la necesidad de invertir un cierto tiempo en la formación de competencias emocionales y, por ello, resulta complejo que se adviertan cambios en un período tan corto como es un curso académico.

Cabe destacar que, en el estudio con alumnos de secundaria, la conciencia emocional tuvo un efecto más llamativo. Este hecho se comprende fácilmente, pues supone un reflejo del proceso de adquisición natural de las competencias emocionales y, su entrenamiento, el cual requiere en primer lugar saber identificar las emociones de uno o de una misma (John y Gross, 2003). En este sentido, algunas investigaciones anteriores han demostrado que constructos similares, como menor claridad emocional, se puede relacionar con las estrategias específicas de regulación de emociones como la supresión. Esto demuestra que una mayor conciencia emocional puede ser beneficiosa para elegir estrategias adaptativas (Arndt, Lischetzke, Crayen y Eid, 2018).

\section{Modelo de competencias emocionales}

En primer lugar, es imprescindible señalar el modelo de competencias emocionales el cual está en constante proceso de construcción, análisis y revisión permanente. (Bisquerra, 2000; Bisquerra y Pérez, 2007). Las competencias emocionales son un aspecto importante de la ciudadanía efectiva y responsable, por eso, el dominio de éstas supone una mejor adaptación al contexto y favorece un afrontamiento más exitoso a las diferentes circunstancias de la vida.

Con el propósito de contribuir al desarrollo de las competencias emocionales, el GROP lleva trabajando desde 1997 la educación emocional tanto en los ámbitos de investigación como en el de la docencia. Se han agrupado en 5 bloques: conciencia emocional, regulación emocional, autonomía emocional, competencia social y habilidades de vida para el bienestar. Se presentan a continuación en forma de pentágono:

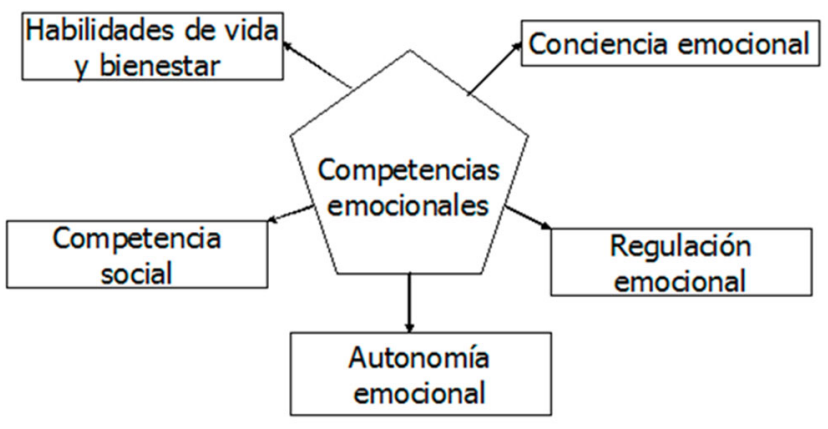

Figura 2.

Modelo de competencias emocionales. Bisquerra (2008).

A continuación, se explica brevemente en que consiste cada competencia (Bisquerra y Pérez-Escoda, 2007):

Conciencia emocional: entendida como la capacidad de tomar conciencia de las propias emociones y de las emociones de los demás, así como captar el clima emocional de un contexto determinado.

Regulación emocional: supone la capacidad para manejar de manera apropiada las emociones, tomando consciencia de la relación entre emoción, cognición y comportamiento. También implica tener buenas estrategias de afrontamiento o generar emociones positivas.

Autonomía emocional: se puede entender como un concepto amplio que incluye un conjunto de características y elementos relacionados con la autogestión personal. Entre ellos se encuentran: la autoestima, actitud positiva ante la vida, responsabilidad, capacidad para analizar críticamente las normas sociales, la capacidad para buscar ayuda y recursos, así como la autoeficacia emocional. 
Competencia social: es la capacidad para mantener buenas relaciones con otras personas. Este hecho implica dominar las habilidades sociales, la capacidad de comunicarse efectivamente, el respeto, la asertividad, las actitudes prosociales, etc.

Competencias para la vida y el bienestar: es la capacidad para adoptar comportamientos apropiados y responsables para afrontar los desafíos diarios de manera satisfactoria, ya sean estos personales, profesionales, familiares, sociales, de tiempo libre, así como organizar nuestra vida de manera sana y equilibrada.

\section{Instrumentos elaborados}

Como señaló Pérez-Escoda (2016), la mayoría de los escasos instrumentos para la evaluación de las competencias emocionales han sido sometidos a críticas centradas en la falta de un marco teórico claro y de fundamentos empíricos firmes (Pérez, Petrides y Furnham, 2005). Para hacer frente a este problema, el GROP ha trabajado intensamente en la elaboración de varios cuestionarios destinados a diferentes franjas de edad. En todos los casos se evalúa la competencia emocional total y sus cinco dimensiones explicadas anteriormente: conciencia emocional, regulación emocional, autonomía emocional, competencia social y competencias para la vida y el benestar.

El cuestionario de Desarrollo emocional de Adultos (versión extensa) CDE-A: Los destinatarios de este cuestionario son adultos (persones mayores de 18 años) con un nivel socioeducativo medio-alto. Es un cuestionario de autoinforme que consta de 48 ítems, de los cuales, 7 elementos miden la conciencia emocional; 13 la regulación emocional, 7 la autonomía emocional, 12 las competencias sociales y 9 las competencias para la vida y el bienestar. Este cuestionario ofrece una puntuación global y otra para cada una de las dimensiones mencionadas, y utiliza una escala de Likert con once opciones de respuesta de cero a diez. Este instrumento ha sido aplicado en diversas investigaciones a profesorado de diferentes niveles educativos y en otros colectivos: profesionales específicos, así como a muestras de estudiantes universitarios. También esta siendo utilizado como instrumento para la evaluación de programas de desarrollo de las competencias emocionales en el marco de algunas tesis doctorales. Algunos trabajos donde se ha empleado este instrumento son: Filella-Guiu, PérezEscoda, Agulló Morera y Oriol (2014), Pérez-Escoda, Filella y Soldevila (2010) o Pérez-Escoda, Filella, Alegre y Bisquerra (2012).

El Cuestionario de Desarrollo Emocional de Adultos (versión reducida) CDE-R: Los estudios realizados con el CDE-A demostraron la importancia de considerar el nivel de estudios debido a las dificultades que se derivan de éste en el momento de la comprensión y el cansancio derivado de la aplicación de este tipo de instrumentos. Concretamente, este instrumento consta de 27 ítems, a la que se denomina CDE-R, destinada a personas adultas (mayores de 18 años) con un nivel medio o inferior de estudios. La elección de los ítems que componen este instrumento se eligieron a partir de la capacidad discriminativa de los ítems del instrumento anteriormente mencionado. Este instrumento ha sido aplicado en diversas investigaciones con personas desempleadas, usuarios de centros cívicos, padres y madres de centros escolares diversos. Algún ejemplo de estos trabajos: Pérez-Escoda y Ribera (2009); o Pérez-Escoda, Velar y Ruiz (2014).

El Cuestionario de Desarrollo Emocional para Educación Secundaria CDE-SEC: este cuestionario dirigido a adolescentes que consiste en una escala de autoinforme que consta de 35 ítems con formato de respuesta tipo Likert, con 11 opciones de respuesta en una escala de 0 (completamente en desacuerdo) a 10 (completamente de acuerdo). Los resultados que se obtienen permiten detectar las necesidades que cada alumno pueda tener a nivel global y en cada una de las dimensiones. Algunos ejemplos de estos trabajos son: García Navarro (2011) Pellicer y Pérez-Escoda (2012). Actualmente también esta siendo utilizado en diversas tesis doctorales.

El Cuestionario de Desarrollo Emocional para Niños CDE9-13: El instrumento orientado a niños y niñas de 9 a 13 años de edad consta de 38 ítems con formato de respuesta tipo Likert de once puntos. Este instrumento ha permitido estudiar las necesidades de desarrollo emocional de niños y niñas de $4^{\circ}$ a $6^{\circ}$ de Educación Primaria. Algunos ejemplos de estos trabajos son: Pérez-Escoda y López-Cassá, 2013) y también se ha utilizado en diversas investigaciones donde se ha observado correlaciones interesantes (Pérez-Escoda, López-Cassà y Torrado, 2012).

\section{A modo de conclusión}

La evidencia científica previa demuestra que las conductas conflictivas tienen una estrecha relación con las competencias emocionales de los alumnos, pues, un peor manejo de las competencias emocionales compete una peor y menos asertiva resolución de conflictos (Bisquerra, 2014; Filella, 2014). Asimismo, se ha demostrado la correspondencia de este hecho con un peor rendimiento escolar y con un estado emocional más vulnerable (Extremera y FernándezBerrocal, 2004c). Por otro lado, se ha comprobado que un mejor manejo de las emociones, así como una autoestima más sana predice claramente un mejor estado emocional de los niños, lo cual se ve reflejado en el clima social de aula y en el rendimiento. Así pues, se pone de manifiesto la relevancia de la educación emocional entendida como un proceso educativo, continuo y permanente, que pretende potenciar el desarrollo de las competencias emocionales como elemento esencial del desarrollo integral de la persona las 
cuales facilitan y predisponen a gozar de una vida más feliz (Bisquerra y Pérez-Escoda, 2007)

Precisamente, las contribuciones presentadas en este artículo persiguen la finalidad de la educación emocional. Concretamente, el videojuego descrito previamente, tiene como meta actuar como estrategia preventiva, incidiendo en los niveles de ansiedad, mejorando el clima tanto en el patio como en el aula, favorecer el rendimiento académico y potenciar la resolución asertiva de conflictos. Los resultados reflejan una mejora de las competencias emocionales de manera estadísticamente significativa, pero cabe destacar la importancia de prolongar esta formación en el tiempo para advertir mayores cambios.

\section{Referencias}

Arndt, C., Lischetzke, T., Crayen, C. y Eid, M. (2018). The assessment of emotional clarity via response times to emotion items: Shedding light on the response process and its relation to emotion regulation strategies. Cognition and Emotion, 32(3), 530-548.

Augustine, A.A. y Hemenover, S.H. (2008). On the relative effectiveness of affect regulation strategies: A metaanalysis. Cognition and Emotion, 23, 1181-1220.

Altarriba, J. y Bauer, L.M. (2004). "The distinctiveness of emotion concepts: A comparison between emotion, abstract, and concrete words". The American Journal of Psychology, 117(3), 389-410. https:// doi.org/10.2307/4149007

Álvarez García, D., Dobarro, A., Álvarez, L., Núñez, J.C. y Rodríguez, C. (2014). La violencia escolar en los centros de educación secundaria de Asturias desde la perspectiva del alumnado. Educación XX1, 17(2), 337-360.

https://doi.org/10.5944/ educxx1.17.2.11494

Baikie, K.A. y Wilhelm, K. (2005). Emotional and physical health benefits of expressive writing. Advances in Psychiatric Treatment, 11(5), 338-346. https:// doi.org/10.1192/apt.11.5.338

Bisquerra, R. (2000). Educación emocional y bienestar. Praxis.

Bisquerra, R. (2008). Ecuación emocional para la convivencia. En: Jiménez Benedit. Educación emocional y convivencia en el aula. Ministerio de Educación, Política social y Deporte.

Bisquerra, R. (2014). Prevención del acoso escolar con educación emocional. Desclée de Brower.

Bisquerra, R. y Pérez-Escoda, N. (2007). Las competencias emocionales. Educación XXI, 10, 61-82.
Bisquerra, R. y Mateo, J. (2019). Competencias emocionales para un cambio de paradigma en educación. Horsori Editorial, S.L.

Butler, E.A., Lee, T.L. y Gross, J.J. (2007). Emotion regulation and culture: Are the social consequences of emotion suppression culture-specific? Emotion, 7 (1), 30-48. https://doi.org/10.1037/15283542.7.1.30

Caballo, V.E. (1997). Manual de evaluación y entrenamiento de las habilidades sociales. Siglo XXI.

Caballo, V., Arias, A., Calderero, M., Salazar, I. y Irurtia, M (2011). Acoso escolar y ansiedad social en niños (I): Análisis de su relación y desarrollo de nuevos instrumentos de evaluación. Behavioral Psychology/ Psicología Conductual, 19(3), 591-609.

Ceballos, E., Correa, N., Correa, A., Rodríguez, J., Rodríguez, B. y Vega, A. (2010). La voz del alumnado en el conflicto escolar. Revista de Educación, 359, 554579. https://doi.org/10-4438/1988-592X-RE-2010359-107.

Conoley, C.W., Conoley, J.C., Mcconnell, J.A. y Kimzey, C.E. (1983). The effect of the ABC's of rational emotive therapy and the empty chair technique of Gestalt therapy on anger reduction. Psychotherapy: Theory, Research, and Practice, 20, 112117. https://doi.org/10.1037/h0088470

Díez, E.J. (2004). Interculturalidad, convivencia y conflicto. Tabanque, 18, pp. 49-76.

Dinkes, R., Kemp, J., Baum, K. y Snyder, T. (2009). Indicators of school crime and safety: (NCES 2010012/NCJ 228478). Washington, DC: National Center for Education Statistics, Institute of Education Sciences, U.S. Department of Education, and Bureau of Justice Statistics, Office of Justice Programs, U.S. Department of Justice.

Elliott, R. y Greenberg, L. (2016). Emotion-focused therapy. In C. Lago y D. Charura (Ed.), The PersonCentred Counselling and Psychotherapy Handbook: Origins, Developments and Current Applications (pp. 212-222). McGrawHill.

Extremera, N. y Fernández-Berrocal, P. (2003). La inteligencia emocional en el contexto educativo: Hallazgos científicos de sus efectos en el aula. Revista de Educación, 332, 97-116.

Extremera, N. y Fernández-Berrocal, P. (2004a). El papel de la inteligencia emocional en el alumnado: evidencias empíricas. Revista electrónica de Investigación Educativa, 6(2) 1-17. 
Extremera, N. y Fernández-Berrocal, P. (2004b). Medidas de evaluación de la inteligencia emocional en el alumnado: evidencias empíricas. Revista latinoamericana de Psicología, 36, 209-228.

Extremera, N. y Fernández-Berrocal, P. (2004c). El uso de las medidas de habilidad en el ámbito de la inteligencia emocional. Boletín de Psicología, 80, 59-77.

Filella, G. (2014). Aprendre a conviure. Barcanova.

Filella, G. (2016). Proceso de la regulación emocional. [Imagen 1]. Recuperado de: https:// repositori.udl.cat/bitstream/handle/10459.1/47639/ gfilellag_proceso_esp.pdf? sequence $=13 \&$ is Allowed $=\mathrm{y}$

Filella-Guiu, G., Pérez-Escoda, N., Agulló, M.J. y Oriol, X. (2014). Resultados de la aplicación de un programa de educación emocional en Educación Primaria. Estudios sobre Educación, 26, 125-147.

Gabarda, V. (2014). Bullying en el aula: Aprende que tipos de bullying existen, cuáles son los factores de riesgo y cómo detectarlo. Universidad Internacional de Valencia, 1, 10-8 .11.

García-Navarro, E. (2011). Competencias emocionales, autoestima y rendimiento académico en adolescentes (Trabajo de Final de Máster). Universidad de Barcelona.

Goleman, D. (1995) Emotional inteligence. Bantam Books.

Gortner, E.M., Rude, S.S. y Pennebaker, J.W. (2006). Benefits of Expressive Writing in Lowering Rumination and Depressive Symptoms. Behavior Therapy, 37(3), 292-303. https://doi.org/10.1016/ j.beth.2006.01.004

Grant, J.E., Odlaug, B.L. y Schreiber, L.N. (2012). Pharmacological Treatments in Pathological Gambling. British Journal of Clinical Pharmacology. https:// doi.org/10.1111/j.1365-2125.2012.04457.x

Gross, J.J. (2002). Emotional regulation: affective, cognitive and social consequences. Psychophysiology, 39 (3), 281-291.

Gross, J.J. (2014). Emotion Regulation: Conceptual and Empirical Foundations. En J. Gross (Ed.). Handbook of emotion regulation (pp. 3-20). Guilford Press.

Gross, J.J. (2015). Emotion Regulation: Current Status and Future Prospects. Psychological Inquiry: An International Journal for the Advancement of Psychological Theory, 26(1), 1-26. https:// doi.org/10.1080/1047840X.2014.940781
Gross, J.J. y Jazaieri, H. (2014). Emotion, Emotion Regulation, and Psychopathology. Clinical Psychological Science, 2(4), 387-401.

Gross, J.J. y John, O.P. (2003). Individual differences in two emotion regulation processes: Implications for affect, relationships, and well-being. Journal of Personality and Social Psychology, 85, 348-362.

Gyurak, A., Gross, J.J. y Etkin, A. (2011). Explicit and implicit emotion regulation: A dual-process framework. Cognition and Emotion, 25, 400-412.

Jeong, H. (2008). Understanding Conflict and Conflict Analysis. SAGE.

John, O.P. y Gross, J.J. (2004). Healthy and unhealthy emotion regulation: Personality processes, individual differences, and life span development. Journal of Personality, 72, 1301-1333. https:// doi.org/10.1111/j.1467-6494.2004.00298.x

Jiménez Benedit, M. (2008). Educación emocional y convivencia en el aula. Ministerio de Educación, Política social y Deporte.

Koole, S.L. (2009): The psychology of emotion regulation: An integrative review. Cognition y Emotion, 23(1), 4-41. https://doi.org/10.1080/02699930802619031

Krumboltz, J.D. (1979). A Social Learning Theory of Career Decision Making. En A. M. Mitchell, G. B. Jones. y J. D. Krumboltz (Eds.). Social Learning and Career Decision Making (pp. 19-50). The Carroll Press.

Lu, Q. y Stanton, A.L. (2010). How benefits of expressive writing vary as a function of writing instructions, ethnicity and ambivalence over emotional expression. Psychology \& Health, 25(6), 669-684. https:// doi.org/10.1080/08870440902883196

McRae, K., Ciesielski, B. y Gross, J. . (2012). Unpacking cognitive reappraisal: Goals, tactics, and outcomes. Emotion, 12(2), 250-255. https:// doi.org/10.1080/01443410.2018.1524851

Monjas, M.I. y González, B.P. (2000). Las habilidades sociales en el currículo. España: Ministerio de Educación, Cultura y Deporte.

Muñíz-Velázquez, J.A. y Álvarez-Nobell, A. (2013). Felicidad, desarrollo de la Cultura en las organizaciones, un enfoque psicosocial. Revista de Comunicación. 12, 7-31.

Narkiss-Guez, T., Enav Zichor, Y., Guez, J. y Diamond, G.M. (2014). Intensifying attachment-related sadness and decreasing anger intensity among individuals suffering from unresolved anger: The role of 
relational reframe followed by empty-chair interventions. Counselling Psychology Quarterly, 28(1), 44-56.

https:// doi.org/10.1080/09515070.2014.924480

Ortega, R. (2010). Agresividad injustificada, bullying y violencia escolar. Alianza Editorial.

Pellicer, I. y Pérez-Escoda, N. (2012). Educació Física emocional. Comunicación presentada en las VIII Jornades d'Educació Emocional: Emocions i salut

Pérez-Escoda, N. (2016). Cuestionarios del GROP para la evaluación de la competencia emocional (CDE). En Soler, Aparicio, Díaz, Escolano y Rodríguez (coords.). Inteligencia emocional y Bienestar II. Reflexiones, experiencias profesionales e investigaciones (pp. 690-705). Universidad San Jorge Ediciones.

Pérez-Escoda, N., Bisquerra, R. Filella, G. y Soldevila, A (2010). Construcción del Cuestionario de Desarrollo Emocional de Adultos (QDE-A). Revista Española de Orientación Psicopedagógica. REOP, 21(2) 367379.

Pérez-Escoda, N., Filella, G., Bisquerra, R. y Alegre, A. (2012). Desarrollo de la competencia emocional de maestros y alumnos en contextos escolares. Electronic Journal of Research in Educational Psychology. 10(3), 1183-1208.

Pérez-Escoda, N., Filella, G., Soldevila, A. y Fondevila, A. (2013). Evaluación de un programa de educación emocional para profesorado de primaria. Educación $X X I, 16,233-254$.

Pérez-Escoda, N., López-Cassà, E. y Torrado, M. (2012). Competencias emocionales y autoestima en Educación Primaria. Comunicación presentada al Congreso Internacional de Docencia Universitaria e Innovación.

Pérez-Escoda, N. y López-Cassà, E. (2013). Relación entre la autoestima, la competencia emocional y el rendimiento académico en los alumnos de la educación Primaria. Comunicación presentada al Congreso Internacional Multidisciplinar de Investigación Educativa: Investigación de calidad para mejorar la educación. Tarragona.

Pérez-Escoda, N. y Ribera, A. (2009). Las competencias emocionales en los procesos de inserción laboral. Revista Española de Orientación y Psicopedagogía, 30(3), 251-256.

Pérez-Escoda, N., Velar, K. y Ruiz, A. (2014). Competencias emocionales y depresión en cuidadores familiares de personas mayores dependientes. Ansiedad y estrés. 20(2-3), 181-191.

Pérez-Fuentes., Gázquez, J., Molero. M. y García (2011). Rendimiento académico y conductas antisociales y delictivas en alumnos de Educación Secundaria Obligatoria. International Journal of Psychology and Psychological Therapy, 11, 401-4012.

Pérez-Gonzalez, J.C, Petrides, K.V y Furnham, A. (2007). La medida de la inteligencia emocional rasgo. En Mestre y Fernández-Berrocal (coords.). Manual de Inteligencia Emocional (pp. 81-97). Pirámide.

Redorta, J., Obiols, M. y Bisquerra, R. (2006). Emoción y conflicto. Aprenda a manejar las emociones. Paidós.

Rimé, B. (2009). Emotion Elicits the Social Sharing of Emotion: Theory and Empirical Review. Emotion Review, 1(1). 60-85. https:// doi.org/10.1177/1754073908097189

Roca. E. (2014). Como mejorar las habilidades sociales. ACDE Ediciones.

Romera, E., Rodríguez, S. y Ortega, R. (2015). Children's perceptions of bullying among peers through the use of graphic representation. Cultura y Educación: Revista de teoría, investigación y práctica, 27(1), 158-185. https:// doi.org/10.1080/11356405.2015.1006850

Rozenblum, S. (2007). Mediación: convivencia y resolución de conflictos en la comunidad. GRAÓ.

Save the Children (2015). Violencia contra la infancia, hacia una Estrategia Integral. Save the Children Recuperado de https://www.savethechildren.es/ publicaciones/violencia-contra-la-infancia-hacia-una -estrategia-integral

Sheppes, G., Scheibe, S., Suri, G., Radu, P., Blechert, J. y Gross, J.J. (2014). Emotion regulation choice: A conceptual framework and supporting evidence. Journal of Experimental Psychology: General, 143 (1), 163-181. https://doi.org/10.1037/a0030831

Stegge, H. y Meerum, M. (2007). Awareness and Regulation of Emotion in Typical and Atypical Development. In J. J. Gross. (Ed.), Handbook of emotion regulation (pp. 269-286). New York, NY: Guilford Press.

Thompson, R.J., Dizén, M. y Berenbaum, H. (2009). The unique relations between emotional awareness and facets of affective instability. Journal of Research in Personality, 43(5), 875-879. https:// doi.org/10.1177/0049124116672678

Villamediana, J., Donado, A. y Zerpa, C.E. (2015). Estilos de manejo de conflictos, inteligencia emocional y 
desarrollo moral. Revista Dimensión Empresarial, 13(1), 73-94. https://doi.org/10.15665/rde.v13i1.339

Wagner-Moore, L.E. (2004). Gestalt Therapy: Past, Present, Theory, and Research. Psychotherapy: Theory, Research, Practice, Training, 41(2), 180189. https://doi.org/10.1037/0033-3204.41.2.180

Webb, T.L., Miles, E. y Sheeran, P. (2012). Dealing with feeling: A meta-analysis of the effectiveness of strategies derived from the process model of emotion regulation. Psychological Bulletin, 138, 775-808.

Webster-Stratton, C. y Reid, M.J. (2004). Strengthening social and emotional competence in young children-The foundation for early school readiness and success: Incredible years classroom social skills and problem-solving curriculum. Infants \& Young Children, 17(2), 96-113.

Werner, K. y Gross. J. (2010). Emotional Regulation and Psychopathology: A Conceptual Framework. En A. M. Kring y D. M. Sloan. (Eds.), Emotional Regulation and Psychopathology A transdiagnostic approach to etiology and treatment (pp. 13-37). The Guilford Press.

Wittmann, M. y Paulus, M.P. (2008). Decision making, impulsivity and time perception. Trends in Cognitive Sciences, 12(1), 7-12. https://doi.org/10.1016/ j.tics.2007.10.004

Zech, E. y Rimé, B. (2005). Is talking about an emotional experience helpful? effects on emotional recovery and perceived benefits. Clinical Psychology \& Psychotherapy, 12(4), 270-287. https:// doi.org/10.1002/cpp.460 